\title{
The Effects of Heart and Skeletal Muscle Inflammation and Cardiomyopathy Syndrome on Creatine Kinase and Lactate Dehydrogenase Levels in Atlantic Salmon (Salmo salar L.)
}

\author{
Muhammad Naveed Yousaf and Mark D. Powell \\ Faculty of Biosciences and Aquaculture, University of Nordland, 8049 Bodo, Norway \\ Correspondence should be addressed to Muhammad Naveed Yousaf, drnavidyusuf@yahoo.com
}

Received 27 January 2012; Accepted 5 March 2012

Academic Editors: G. C. David, D. Endoh, and S. W. Feist

Copyright (C) 2012 M. N. Yousaf and M. D. Powell. This is an open access article distributed under the Creative Commons Attribution License, which permits unrestricted use, distribution, and reproduction in any medium, provided the original work is properly cited.

\begin{abstract}
Heart and skeletal muscle inflammation (HSMI) and cardiomyopathy syndrome (CMS) are putative viral cardiac diseases of Atlantic salmon. This study examined the levels and correlated the serum enzymes creatine kinase (CK) and lactate dehydrogenase (LDH) to the histopathology of clinical outbreaks of HSMI and chronic CMS in farmed Atlantic salmon. A total of 75 fish from 3 different HSMI outbreaks, 30 chronic CMS fish, and 68 fish from 3 nondiseased fish groups were used as the study population $(N=173)$. Serum CK and LDH levels correlated significantly with the total inflammation and total necrosis scores for HSMI fish $(P=0.001)$. However, no correlation was identified for enzyme levels and histopathology scores for chronic CMS fish. The significantly increased CK and LDH levels and their positive correlations to histopathology differentiate HSMI from CMS clinically suggesting the potential use of enzymes for screening for HSMI is promising.
\end{abstract}

\section{Introduction}

The marine farmed Atlantic salmon (Salmo salar L.) exhibits a variety of cardiac diseases, and the reason for this likely includes low activity in relatively confined spaces, continuous food supply, low oxygen level, crowding, stress in handling, and temperature [1]. The cardiac anomalies and defects of Atlantic salmon include aplasia or hypoplasia of the septum transversum, abnormal location and shape of heart [1], arteriosclerosis $[2,3]$, and ventricular hypoplasia [4], but specific diseases include cardiomyopathy syndrome (CMS) [5-7], pancreas disease (PD) [8-10], and heart and skeletal muscle inflammation (HSMI) [11, 12]. Annual economical losses due to cardiomyopathy syndrome (CMS) alone have been estimated up to $€ 4$ 4.5-8.8 millions [7].

Heart and skeletal muscle inflammation (HSMI) is a disease of marine farmed Atlantic salmon reported from Norway, Scotland and Chile. HSMI is a disease which mainly affects heart and red skeletal muscle. It is typically a disease of moderate mortality $(\sim 20 \%)$ but high morbidity $(\sim 100 \%)$ that affects fish 5 to 9 months after transfer to sea. Presently,
HSMI can be diagnosed by histopathology and presents as epi- and endocarditis as well as mononuclear cellular infiltration of both trabecular and compact layers of ventricle myocardium accompanied by myocytic necrosis [11-14]. HSMI is transmissible in laboratory studies by injecting tissue homogenate from diseased fish to healthy fish $[11,15]$, and recently piscine reovirus (PRV) has been suggested to be associated with HSMI infection [16, 17]. Lesions first appear and are more frequent in heart than red skeletal muscle. Affected myocytes show signs of degeneration, loss of cardiomyocytes striation and eosinophilia, loss of skeletal muscle striation, vacuolation, centralized nuclei, and karyorrhexis. There are more inflammatory changes as compared to necrotic changes in heart and red skeletal muscle $[1,12,13]$. HSMI has become more significant where outbreaks have increased from 54 in 2004 [18] to 162 cases reported in 2011 [19].

Cardiomyopathy syndrome (CMS) is a cardiac disease of Atlantic salmon with a suggested totiviral etiology [20] that mainly affects atrium and trabecular ventricle myocardium without involvement of skeletal muscle. It shares similar 
features with HSMI where both cause myocarditis [1]. Histopathological changes include necrosis and inflammation of trabecular layer of ventricle and atrium, epicarditis, cellular infiltrates of mainly mononuclear lymphocytes and macrophages, and rupture of atrium or sinus venosus macroscopically [1, 5]. CMS affects adult salmon after 12-18 months of sea transfer, and recently a totivirus (piscine myocarditis virus (PMCV)) is proposed as causative agent for cardiomyopathy $[5,17,20,21]$. The piscine myocarditis virus is a double-stranded RNA virus with diameter of $50 \mathrm{~nm}$ and $6688 \mathrm{bp}$ genome size [21]. The haematological tests and serum analysis for fish, compared with other areas of veterinary medicine, are not common place compared to higher vertebrates due to the lack of reference values for clinical chemistry (physiological and pathological) and understanding of disease pathogenesis and pathophysiology [22-24]. There is a strong need for blood biochemistry ranges especially where the etiology is unclear (HSMI and CMS) since the associated viruses can be present asymptomatically $[17,25]$. Biochemical enzymes such as creatine kinase (CK) and lactate dehydrogenase (LDH) are wellestablished biomarkers of cardiac disease in humans [26] and are often used in conjunction with other hormonal biomarkers for a myocardial dysfunction diagnosis [27]. Both enzymes are released upon cellular degeneration such as necrosis. Lactate dehydrogenase $(\mathrm{LDH})$ is involved in the interconversion of pyruvate and L lactate during the final reactions of glycolysis and is present in the cytoplasm of all cells (nucleated and nonnucleated cells). In humans, raised LDH plasma values are observed from 8 to $12 \mathrm{~h}$, peaking within 2-3 days, and levels are sustained for duration of 7-10 days following cardiac injury [28]. Creatine kinase (CK), on the other hand, is found in the myocyte cytoplasm, sarcoplasmic reticulum, mitochondria, and myofibrils with a half-life of about $12 \mathrm{~h}$ in humans. Creatine kinase levels in blood plasma rise from 4 to $6 \mathrm{~h}$ peaking at $12-36 \mathrm{~h}$ and sustained over 3-4 days in humans where a cardiac injury has occurred [28]. The creatine kinase concentrations are related to the irreversible injury associated with myocardial necrosis in mammals (dogs) [29]. The elevated CK levels have been reported in association with myocytic necrosis seen in pancreas disease (PD) in Atlantic salmon [30, 31], and these results suggested that $\mathrm{CK}$ could be a useful candidate indicator of cardiac diseases in Atlantic salmon.

The aims of the present study were to measure the serum $\mathrm{CK}$ and LDH levels and examining their relationship to the cardiac diseases (HSMI and CMS) of Atlantic salmon.

\section{Material and Methods}

2.1. Fish Sampling. Seven groups of Atlantic salmon (Salmo salar L.) were involved in this study. All samples from diseased fish were taken from the farms diagnosed with both diseases by National Veterinary Institute, Oslo, Norway (NVI) and further histopathology was performed to confirm the disease diagnosis during the study. Group $1(n=28)$ consisted of fish (S1) collected from a confirmed HSMI outbreak at a sea site during the peak mortality of the HSMI episode. Group $2(n=16)$ comprised fish (S0) collected from a confirmed HSMI sea cage outbreak two months after peak mortality period. Group $3(n=31)$ included fish (S0) from a sea cage site collected during the early onset of a HSMI outbreak. The clinical phase of the disease has been defined as the time period with increased mortality at farm due to HSMI [32]. The disease phase was determined from the peak mortality time at the farm [16]. All diseased groups were in the sea phase of salmon production and opportunistic samples collected from diseased cages on each farm. All three diseased farms were widely distant from each other in Nordland county, Norway. Group $4(n=30)$ included chronic CMS infected fish (S2) and had confirmed CMS outbreak in the past, and sampling was performed 6 months after CMS outbreak. Diseased groups included fish with average weight range (600-1000 g) for HSMI and (6000$7000 \mathrm{~g})$ for CMS fish.

Group $5(n=28)$ was nondiseased fish which were taken from a study where Atlantic salmon (S0) had been made anaemic using phenylhydrazine, and cardiac hypertrophy had been characterized [33]. Group $6(n=20)$ consisted of nondiseased, apparently healthy fish $(\mathrm{S} 0+\mathrm{S} 1)$ kept in a laboratory facility (University of Nordland, Mørkvedbukta Research Station, Bodo, Norway) in $2 \mathrm{~m}^{3}$ tanks with fresh ambient sea water (temperature range $7-8^{\circ} \mathrm{C}$ ) and fed $0.7 \%$ commercial feed (Spirit, Skretting, Stavanger, Norway) of their body weight three times weekly. Group 7 ( $n=20)$ comprised of apparently healthy, nondiseased Atlantic salmon (S1) from the sea cages. Nondiseased groups included fish with average weight range (400-2000 g).

2.2. Blood Collection. All fish were killed by a blow to the head or overdose of tricaine methanesulfonate (MS222) $\left(100 \mathrm{mg} \mathrm{mL}^{-1}\right)$. Blood was collected immediately from the caudal vein with a $5 \mathrm{~mL}$ syringe using $23 \mathrm{G}$ needle, allowed to clot in Eppendorf tubes for 2-4 h, centrifuged at 8,000 g for $5 \mathrm{~min}$, and the serum collected except group 4 where heparinised blood plasma was collected and frozen at $-20^{\circ} \mathrm{C}$.

2.3. Serum Analysis. All samples were frozen and sent on dry ice to Norwegian School of Veterinary Sciences, Oslo Central Laboratory and to the Nordland Hospital, Department of Medicine Biochemistry, Bodo for creatine kinase (CK) and lactate dehydrogenase (LDH) analysis. Creatine kinase (CK) and lactate dehydrogenase $(\mathrm{LDH})$ were measured by using ADIVA 1650 (Siemens Medical Solution Diagnostics Inc., Tarrytown, NY, USA) at Norwegian school of veterinary sciences, Oslo central laboratory and ADVIA 1650/1800 (Bayer Diagnostics, Tarrytown, NY, USA) at Nordland Hospital, Department of Medicine Biochemistry, Bodo on the basis of their enzyme activity and measured by increase in absorbance at $340 / 410 \mathrm{~nm}$. Both laboratories used the same methods. Briefly the principle of the procedures for $\mathrm{LDH}$ and $\mathrm{CK}$ is as follows: LD catalyzes the conversion of $\mathrm{L}$ lactate to pyruvate in the presence of nicotinamide adenine dinucleotide (NAD). The enzyme activity of LD is proportional to the rate of production of $\mathrm{NADH}$ (reduced NAD). Creatine kinase reacts with creatine phosphate and 
ADP to form ATP which is coupled to the hexokinase-G6PD reaction, generating NADPH. The concentrations of $\mathrm{NADH}$ and NADPH were measured by the increase in absorbance at 340/410 $\mathrm{nm}$ for $\mathrm{LDH}$ and CK, respectively.

2.4. Histopathology and Scoring Method. To correlate biochemical enzymes (CK and LDH), histopathology was method of choice for diagnosis of HSMI. Hearts, skeletal red and white muscle from below the dorsal fin and above the lateral line, and other vital organs, were collected and fixed in $10 \%$ neutral phosphate-buffered formalin solution. External and internal visual examination was performed in addition to histological observation of other vital organs for other abnormalities or signs of overt disease in the fish. Tissues were processed by a standard paraffin wax protocol (dehydrated, embedded in paraffin, $3 \mu \mathrm{m}$ thick sectioned and $\mathrm{H}$ and $\mathrm{E}$ stained) and examined for changes (necrosis and inflammation) characteristic of HSMI [11]. The case definition for HSMI includes inflammation and necrosis of trabecular and compact ventricle myocardium, epicarditis, endocarditis, mononuclear inflammatory cell infiltration, and a higher level of inflammation compared with necrosis while supportive signs may also include inflammation and necrosis of red skeletal muscle, atrium, and absence of pancreatic lesions [32]. A semiquantitative assessment of each slide was adapted from McLoughlin et al. [34] for scoring histopathological findings in heart and muscle tissues obtained from seven groups of fishes used in this study. It has been used and established in PD, a similar cardiac disease to HSMI and CMS (Table 1).

2.5. Different Anatomical Regions. The scoring method was used for the detailed study of the tissue in anatomically distinct areas of the heart and skeletal muscle: (1) atrial trabecular myocardial inflammation, (2) and necrosis, (3) atrial epicarditis, (4) ventricle compact myocardial inflammation, (5) and necrosis, (6) ventricle trabecular myocardial inflammation, (7) and necrosis, (8) ventricle epicarditis, (9) skeletal muscle inflammation, (10) and necrosis. A total inflammation score was determined from the summed scores of all parameters (atrial and ventricular trabecular inflammation, atrial and ventricular epicarditis, ventricle compact inflammation, and skeletal muscle inflammation) and total necrosis score determined from the summed scores of all parameters (atrial and ventricular trabecular necrosis, ventricle compact necrosis, and skeletal muscle necrosis). The sum scores of inflammation and necrosis in heart and skeletal muscle were correlated with the biochemical enzymes (CK and LDH) levels. Total inflammation and total necrosis scores were correlated to the HSMI plus nondiseased fish and the CMS fish plus nondiseased fish to differentiate the enzymatic effects in each disease separately.

2.6. Slides Evaluation. Slides were evaluated blindly by two persons, histopathological results compared between the groups and correlated with respective biochemical enzymes values for each sample. Sometimes the conflict for slide score was around $0.5-1$ between two persons, and then they agreed
TABLe 1: Semiquantitative lesion scoring system adapted from McLoughlin et al. [34]. System covers heart and skeletal muscle lesions separately. Lesions starting from 0 (healthy tissue) to 3 (severe changes). (a) Heart lesion classification. (b) Skeletal muscle classification.

\begin{tabular}{|c|c|}
\hline \multicolumn{2}{|c|}{ Score Description } \\
\hline \multicolumn{2}{|c|}{ (a) } \\
\hline 0 & Normal appearance \\
\hline 1 & Focal myocytic necrosis \pm inflammation $(<50$ fibers affected $)$ \\
\hline 2 & $\begin{array}{l}\text { Multifocal myocytic necrosis } \pm \text { inflammation ( } 50-100 \text { fibers } \\
\text { affected) }\end{array}$ \\
\hline 3 & $\begin{array}{l}\text { Severe diffuse myocytic necrosis } \pm \text { inflammation }(>100 \\
\text { fibers affected) }\end{array}$ \\
\hline \multicolumn{2}{|c|}{ (b) } \\
\hline 0 & Normal appearance \\
\hline 1 & Focal myocytic necrosis \pm inflammation \\
\hline 2 & Multifocal myocytic necrosis \pm inflammation \\
\hline 3 & Severe diffuse myocytic necrosis \pm inflammation \\
\hline
\end{tabular}

after discussing case definition and scoring system on most suitable score for the slide.

2.7. Statistical Analysis. Spearman rank coefficient correlations were performed using SigmaPlot (10.0) and were considered statistically significant at $P$ values $\leq 0.05$. The mean histopathology (total inflammation and total necrosis scores) and enzyme (CK and LDH) values were analyzed using Kruskal-Wallis One Way Analysis of Variance on ranks with differences isolated using Dunn's post hoc analysis. Data were presented as mean $\pm \mathrm{SD}$.

\section{Results}

The highest and lowest mean CK values were identified in HSMI fish group $1=16479.25 \pm 1844.49$ IU.L ${ }^{-1}$ and nondiseased fish group $5=1581.71 \pm 425.33$ IU.L $^{-1}$ respectively. However, the highest and lowest mean $\mathrm{LDH}$ values were identified in HSMI fish group $2=1838.25 \pm 957.47$ IU.L ${ }^{-1}$ and nondiseased fish group $5=235.39 \pm 27.43$ IU.L ${ }^{-1}$, respectively. The mean $\mathrm{CK}$ and $\mathrm{LDH}$ values for CMS fish were $\left(5207.93 \pm 967.81\right.$ IU.L $\left.^{-1}\right)$ and $\left(426.2 \pm 60.68\right.$ IU.L $\left.{ }^{-1}\right)$, respectively (Table 4 ).

Diseased group 1 (HSMI fish) had significantly the highest levels for CK activity as compared to other HSMI groups 2 and 3 (Kruskal-Wallis One-Way Analysis: $\mathrm{H}=$ 65.217, d.f. $=6 ;(P \leq 0.001)$ (Figure 2(a)). However, LDH activity levels were higher in HSMI fish (group 3) as compared to other HSMI fish (groups 1 and 2) (KruskalWallis One-Way Analysis: $\mathrm{H}=73.838$, d.f. $=6 ; P \leq 0.001$ ) (Figure 2(b)). The CMS (group 4) CK and LDH values were significantly different from nondiseased fish (groups 5, 6, and 7) (Figures 2(a) and 2(b)).

The scoring grades which were used to score the inflammatory changes represented by micrographs (Figure 1) and reflected the semiquantitative scoring system which was 


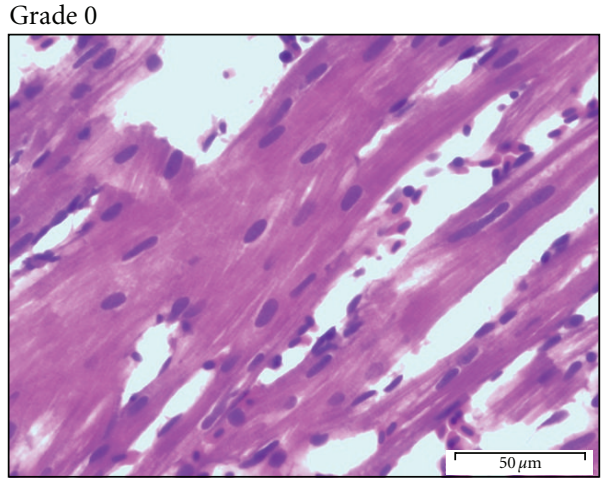

(a)

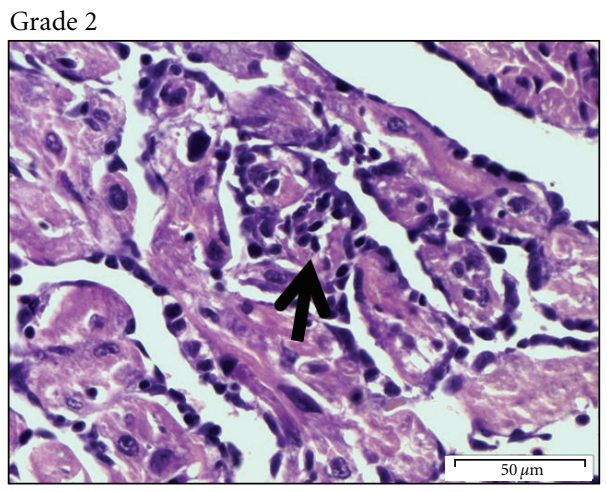

(c)

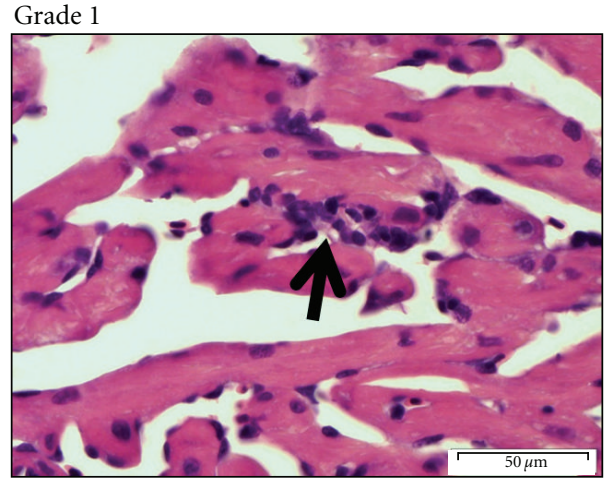

(b)

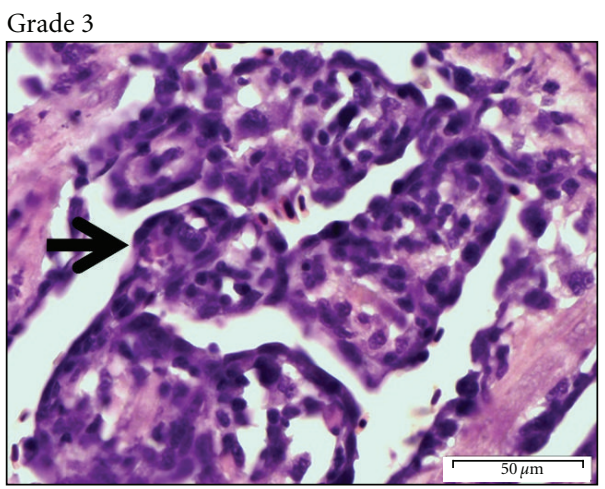

(d)

FIGURE 1: The representative micrographs of the semiquantitative scoring system described in Table 1, represented the heart histopathology. Grade 0: no pathological changes. Grade 1: minor inflammatory lesions comprises of focal subendocardial mononuclear leukocytes. Grade 2: several distinct lesions with moderately increased number of mononuclear leukocytes. Grade 3: severe lesions where almost all myofibres have been replaced by inflammatory cells, predominantly by mononuclear lymphocyte-like cells. Arrow: inflammation. Scale bars $=50 \mu \mathrm{m}$.

TABle 2: Total mean ( \pm SD) scores of inflammation and necrosis for HSMI (groups 1, 2, and 3), CMS (group 4), and nondiseased fish (groups 5, 6, and 7) in heart and heart with skeletal muscle. Different letters represent significant differences between groups $(P<0.05)$.

\begin{tabular}{lccc}
\hline Parameter & Nondiseased & HSMI & CMS \\
\hline Inflammation & & & \\
$\quad$ Heart & $0.83 \pm 0.30$ & $5.30 \pm 1.16$ & $2.44 \pm 1.39$ \\
$\quad$ Heart + muscle & $0.99 \pm 0.16^{\mathrm{a}}$ & $5.59 \pm 1.03^{\mathrm{b}}$ & $2.44 \pm 1.39^{\mathrm{c}}$ \\
\hline Necrosis & & & $0.1 \pm 0.40$ \\
Heart & $0.35 \pm 0.36$ & $2.01 \pm 1.34$ & $0.38 \pm 0.68^{\mathrm{c}}$ \\
$\quad$ Heart + muscle & $0.62 \pm 0.45^{\mathrm{a}, \mathrm{c}}$ & $2.92 \pm 1.15^{\mathrm{b}}$ & \\
\hline
\end{tabular}

applied to each anatomical region of the heart (atrium, compact and trabecular ventricular myocardium, pericardium) and the red skeletal muscle (Table 1).

Seven fish groups were compared on the basis of inflammation and necrosis scores. Inflammatory mononuclear cells were more frequent as compared to focal areas of necrosis in heart tissue, but the opposite was apparent in the red skeletal muscle where necrosis predominated. The mean total inflammation (Kruskal-Wallis One-Way Analysis: $\mathrm{H}=111.216$, d.f. $=2 ;(P \leq 0.001)$ and total necrosis (Kruskal-Wallis One-Way Analysis: $\mathrm{H}=90.484$, d.f. $=2$; $(P \leq$ 0.001 ) scores of HSMI and CMS fish were significantly different from nondiseased fish with the exception of total necrosis scores of CMS fish which were not significantly different from nondiseased fish (Table 2). More lesions were present in the heart as compared to the skeletal muscle (Table 2). Total inflammation scores ranged from 1 to 11 in diseased groups while 0 to 3.5 in nondiseased groups. Total necrosis scores ranged from 0 to 7 for diseased groups while from 0 to 3 for nondiseased groups. Total necrosis (KruskalWallis One-Way Analysis: $\mathrm{H}=118.135$, d.f. $=6 ;(P \leq$ 0.001) (Figure 3(b)) and total inflammation (Kruskal-Wallis One-Way Analysis: $\mathrm{H}=119.558$, d.f. $=6 ;(P \leq 0.001)$ (Figure 3(a)) scores were compared for all seven groups and identified higher scores in HSMI group 1 while being significant and lower in the group 2 and 3 as compared 


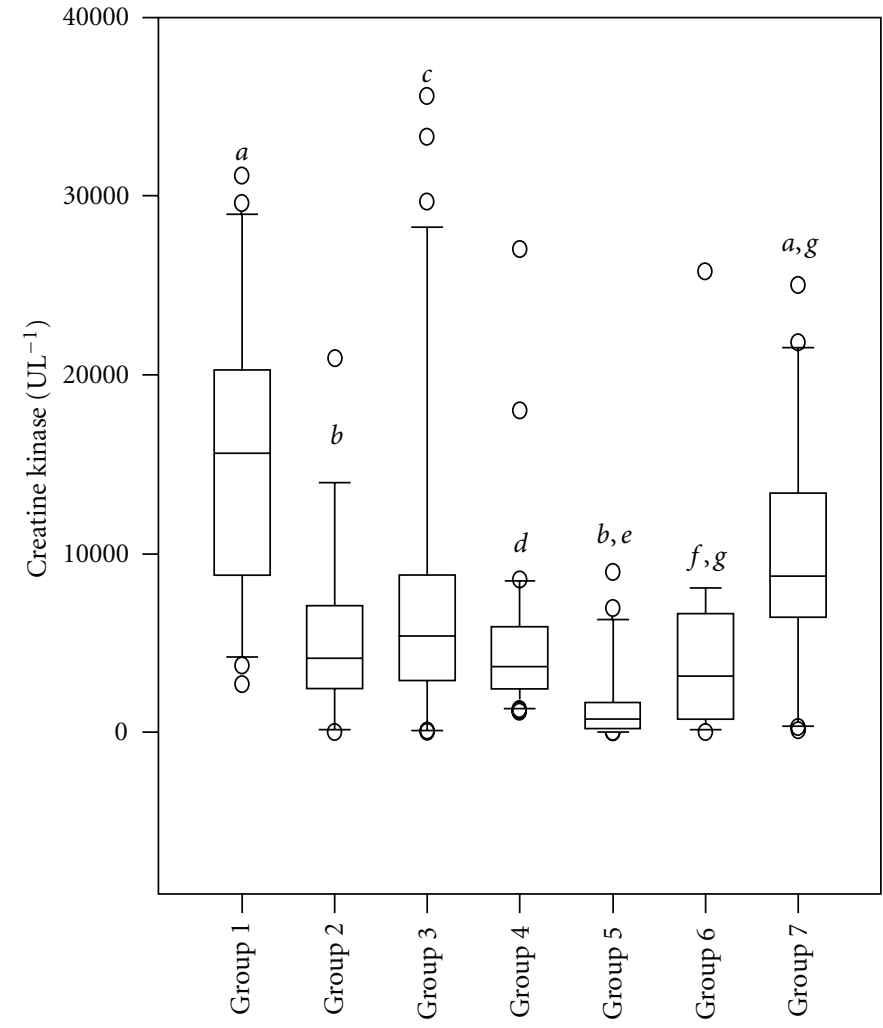

(a)

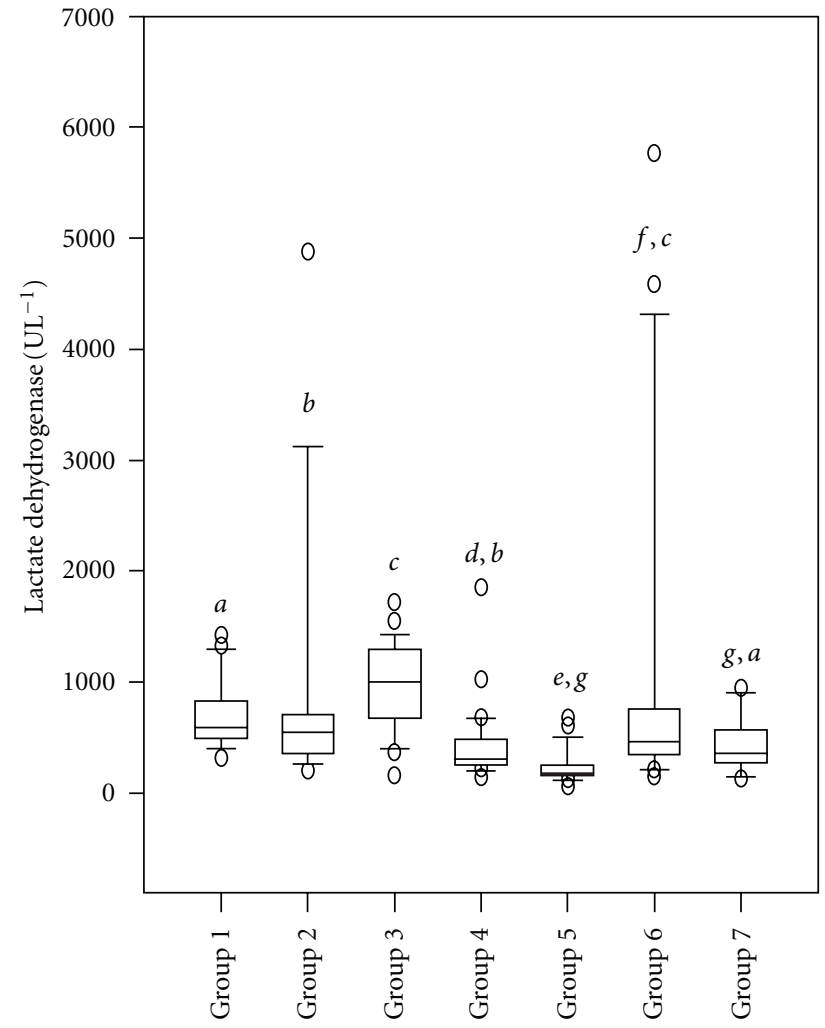

(b)

FIgURe 2: The box plots of (a) creatine kinase (CK) and (b) lactate dehydrogenase (LDH) enzymes activity values in the serum/plasma of Atlantic salmon for all seven groups $(N=173)$. Group $1(n=28)$ and group $2(n=16)$ represented fish from an acute and late phase of a HSMI outbreak, respectively, while group $3(n=31)$ represented values from fish from an early phase of HSMI. Group 4 ( $n=30)$ included chronic CMS fish. Group $5(n=28)$, group $6(n=20)$, and group $7(n=20)$ represented values from nondiseased fish. Bars with different letters represented significant differences between groups $(P<0.05)$. ( $\circ)$ denotes outliers.

to none to low level of changes seen in nondiseased fish (groups 5, 6, and 7) (Figures 3(a) and 3(b)). In general, muscle necrosis and inflammation (cardiac and skeletal) were negligible to mild in nondiseased fish (groups 5, 6, and 7 ) as compared to CMS and HSMI fish (groups 1, 2, 3, and 4). Total inflammation and total necrosis results of all HSMI-diseased fish (groups 1, 2, and 3) were significantly different from nondiseased groups (groups 5, 6, and 7) with the exceptions of total necrosis scores of CMS fish (group 4) which was not significantly different from nondiseased fish (group 6) (Figures 3(a) and 3(b)).

The histopathology scores (sum score of heart and muscle necrosis and inflammation) were correlated with serum/plasma CK and LDH levels. The correlations which were made among CK enzyme levels, and different anatomical parameters of all fish groups excluding CMS fish (group $4)$ gave significant relationships $(P \leq 0.001)$ (Table 3$)$. The significant correlations of CK levels to individual parameters included atrial inflammation ( $\mathrm{S}$ coeff. $=0.451, P<0.001$ ), atrial necrosis ( $\mathrm{S}$ coeff. $=0.252, P=0.002)$, atrial epicarditis (S coeff. $=0.314, P<0.001)$, ventricle compact layer inflammation ( $\mathrm{S}$ coeff. $=0.440, P<0.001)$, ventricle compact layer necrosis ( $\mathrm{S}$ coeff. $=0.249, P=0.002$ ), ventricle trabecular inflammation ( $\mathrm{S}$ coeff. $=0.526, P<0.001$ ), ventricle trabecular necrosis (S coeff. $=0.283, P<0.001$ ), ventricle epicarditis ( $\mathrm{S}$ coeff. $=0.333, P<0.001$ ), skeletal muscle necrosis ( $\mathrm{S}$ coeff. $=0.206, P=0.035$ ), and skeletal muscle inflammation ( $\mathrm{S}$ coeff. $=0.169, P=0.084$ ). The CK enzyme levels significantly and positively correlated with the both total inflammation ( $\mathrm{S}$ coeff. $=0.552, P<0.001$ ) and total necrosis ( $\mathrm{S}$ coeff. $=0.526, P<0.001)$ scores (Table 3 ). The $\mathrm{LDH}$ levels were also correlated in the same manner as above for CK which showed significant relationships $(P=0.05)$ (Table 3). The significant correlations for LDH levels to different parameters were atrial inflammation ( $S$ coeff. $=0.254$, $P=0.002)$, ventricle compact layer inflammation ( $\mathrm{S}$ coeff. $=$ $0.297, P=0.001$ ), ventricle trabecular inflammation (S coeff. $=0.166, P=0.049)$, skeletal muscle inflammation ( $\mathrm{S}$ coeff. $=$ $0.373, P=0.001$ ), ventricle epicarditis ( $\mathrm{S}$ coeff. $=0.20, P=$ 0.016 ), and skeletal muscle necrosis ( $\mathrm{S}$ coeff. $=0.414, P=$ 0.001 ) (Table 3). However, few non-significant relationships were identified for $\mathrm{LDH}$ and anatomical parameters such as ventricle compact layer necrosis ( $\mathrm{S}$ coeff. $=0.080, P=$ 0.341 ), ventricle trabecular necrosis ( $\mathrm{S}$ coeff. $=0.052, P=$ 0.534 ), atrial necrosis ( $\mathrm{S}$ coeff. $=0.034, P=0.688$ ), and atrial epicarditis ( $\mathrm{S}$ coeff. $=-0.049, P=0.558$ ). There were significant positive correlations between LDH levels and the total inflammation $(\mathrm{S}$ coeff. $=0.266, P<0.001$ ) 


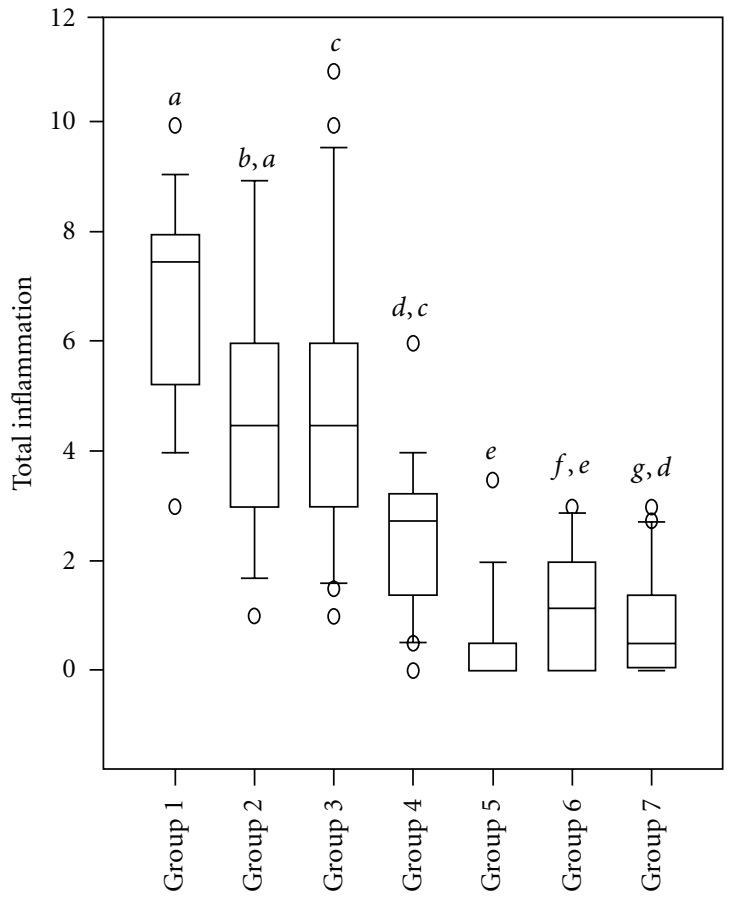

(a)

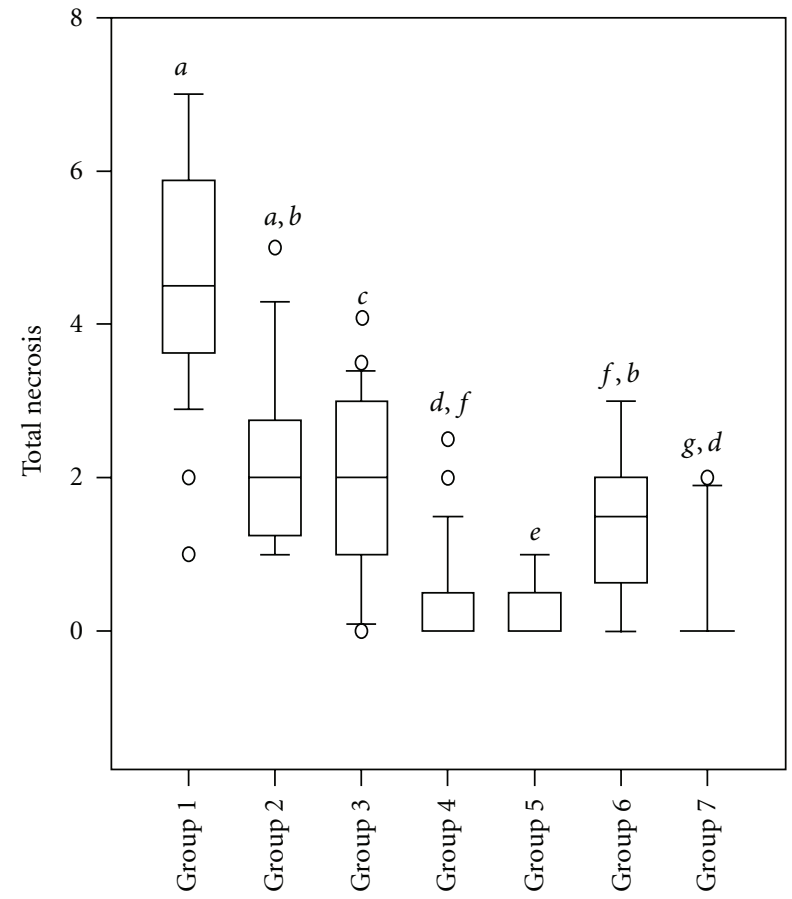

(b)

FIGURE 3: The box plots of all seven groups of Atlantic salmon on the basis of (a) total inflammation and (b) total necrosis in the heart and skeletal muscle $(N=173)$. Group $1(n=28)$ and group $2(n=16)$ represented fish from an acute and late phase of a HSMI outbreak, respectively, while group $3(n=31)$ represented values from fish from an early phase of HSMI, and group $4(n=30)$ included chronic CMS fish. Group $5(n=28)$, group $6(n=20)$, and group $7(n=20)$ represented values from nondiseased (non-HSMI) fish. Higher scores of inflammation and necrosis were found in diseased (groups 1, 2, 3, and 4) fish while low levels of scores in nondiseased (groups 5, 6, and 7) fish were present. Bars with different letters represented significant differences between groups $(P<0.05)$. ( $\circ)$ denotes outliers.

TABLE 3: The Spearman correlation coefficient for anatomically distinct regions of the HSMI and CMS infected fish to creatine kinase (CK) and lactate dehydrogenase (LDH). $P$ values given in parentheses.

\begin{tabular}{|c|c|c|c|c|}
\hline \multirow{2}{*}{ Parameter } & \multicolumn{2}{|c|}{ HSMI } & \multicolumn{2}{|c|}{ CMS } \\
\hline & $\mathrm{CK}$ & $\mathrm{LDH}$ & $\mathrm{CK}$ & $\mathrm{LDH}$ \\
\hline Ventricle compact necrosis & $0.249(0.002)$ & $0.080(0.341)$ & - & - \\
\hline Ventricle trabecular necrosis & $0.283(<0.001)$ & $0.052(0.534)$ & $0.198(0.291)$ & $0.122(0.516)$ \\
\hline Atrium necrosis & $0.252(0.002)$ & $0.034(0.688)$ & $0.327(0.077)$ & $0.242(0.195)$ \\
\hline Skeletal muscle necrosis & $0.206(0.035)$ & $0.414(0.001)$ & $0.068(0.719)$ & $-0.024(0.899)$ \\
\hline Ventricle compact inflammation & $0.440(<0.001)$ & $0.297(0.001)$ & - & - \\
\hline Ventricle trabecular inflammation & $0.526(<0.001)$ & $0.166(0.049)$ & $0.157(0.405)$ & $-0.043(0.822)$ \\
\hline Atrial inflammation & $0.451(<0.001)$ & $0.254(0.002)$ & $0.108(0.580)$ & $0.052(0.790)$ \\
\hline Ventricle epicarditis & $0.333(<0.001)$ & $0.20(0.016)$ & $0.340(0.065)$ & $0.309(0.096)$ \\
\hline Atrium epicarditis & $0.314(<0.001)$ & $-0.049(0.558)$ & - & - \\
\hline Muscle inflammation & $0.169(0.084)$ & $0.373(0.001)$ & - & - \\
\hline Total inflammation & $0.552(<0.001)$ & $0.266(<0.001)$ & $0.089(0.635)$ & $-0.075(0.691)$ \\
\hline Total necrosis & $0.526(<0.001)$ & $0.247(0.003)$ & $0.355(0.075)$ & $0.240(0.209)$ \\
\hline
\end{tabular}

and total necrosis (S coeff. $=0.247, P<0.003$ ) scores (Table 3). The correlations between CK enzyme levels and different anatomical parameters of all fish groups excluding HSMI fish (groups 1, 2, and 3) identified non-significant relationships $(P=0.05)$ (Table 3$)$. The correlations made between $\mathrm{CK}$ levels and different anatomical regions were atrial inflammation ( $\mathrm{S}$ coeff. $=0.108, P=0.580$ ), atrial necrosis ( $\mathrm{S}$ coeff. $=0.327, P=0.077$ ), ventricle trabecular inflammation (S coeff. $=0.157, P=0.405)$, ventricle trabecular necrosis ( $\mathrm{S}$ coeff. $=0.198, P=0.291$ ), ventricle epicarditis ( $\mathrm{S}$ coeff. $=0.340, P=0.065$ ), and skeletal muscle necrosis $(\mathrm{S}$ coeff. $=0.068, P=0.719)$. The LDH levels 
TABLE 4: Blood serum enzymes in different fish groups (Mean \pm SE).

\begin{tabular}{lcc}
\hline & Creatine kinase $\left(\mathrm{IU} . \mathrm{L}^{-1}\right)$ & ${\text { Lactate dehydrogenase }\left(\mathrm{IU} . \mathrm{L}^{-1}\right)}$ \\
\hline Group 1 & $16479.25 \pm 1844.49$ & $697.43 \pm 56.61$ \\
Group 2 & $10280 \pm 5246.18$ & $1838.25 \pm 957.47$ \\
Group 3 & $8333.34 \pm 1709.08$ & $966 \pm 71.94$ \\
Group 4 & $5207.93 \pm 967.81$ & $426.2 \pm 60.68$ \\
Group 5 & $1581.71 \pm 425.33$ & $235.39 \pm 27.43$ \\
Group 6 & $7098.35 \pm 2916.95$ & $1027.9 \pm 334.04$ \\
Group 7 & $10297.15 \pm 1531.11$ & $423.3 \pm 53.06$ \\
\hline
\end{tabular}

were also correlated in the same manner as above for CK and included atrial inflammation (S coeff. $=0.052, P=$ 0.790 ), atrial necrosis ( $\mathrm{S}$ coeff. $=0.242, P=0.195$ ), ventricle trabecular inflammation ( $\mathrm{S}$ coeff. $=-0.043, P=0.822)$, ventricle trabecular necrosis ( $\mathrm{S}$ coeff. $=0.122, P=0.516$ ), ventricle epicarditis ( $\mathrm{S}$ coeff. $=0.309, P=0.096$ ), and skeletal muscle necrosis ( $\mathrm{S}$ coeff. $=-0.024, P=0.899$ ). The combined CMS and nondiseased group's correlations with CK levels were also made in the same manner as described above and identified correlations for total inflammation (S coeff. $=0.089, P=0.635)$ and total necrosis ( $\mathrm{S}$ coeff. $=$ $0.355, P=0.075$ ) scores (Table 3 ). There were non-significant negative and positive correlations between LDH levels and total inflammation and total necrosis for CMS group, respectively (Table 3 ).

\section{Discussion}

The CK and LDH values of all seven fish groups were compared and diseased fish (HSMI) identified with significantly higher enzymes levels as compared to nondiseased fish. The significantly higher and lower mean enzymes levels in diseased and nondiseased fish, respectively, were consistent with the CK enzyme ranges already reported in farmed Atlantic salmon affected with a similar pancreas disease (PD) $[30,31]$. The highest mean LDH levels were identified at the earlier phase of HSMI disease while highest CK levels were present in acute phase of the disease. Previous in vivo studies identified the increased CK and LDH activities in Atlantic salmon and Nile tilapia (Oreochromis niloticus) treated with tributyltin (TBT) and cadmium, respectively $[35,36]$.

Histopathology was used as a method of choice to diagnose the diseases (HSMI and CMS). This study described the histopathology in the heart and skeletal muscle by using a semiquantitative scoring system that addressed the pathological changes in both tissues (cardiac and somatic muscle). The diseased fish showed the histopathological changes in the heart and skeletal muscle similar to HSMI and in hearts for CMS fish $[5,11,13]$. The histological changes were identified in both atrium and ventricle (compact and trabecular) of HSMI fish while mostly ventricular trabecular layer was involved in CMS fish. The histopathological changes were compared for all seven fish groups, and hearts were identified with most tissue damage and suggested to be the contributing source of enzymes (CK and LDH) which released upon cellular damage and in line with Rodger et al. [30] that suggested the significantly higher CK levels due to myopathy in PD-affected Atlantic salmon.

The mean CK levels and histopathology scores for acute phase HSMI fish (group 1) were doubled than early or late phase of HSMI fish (groups 2 and 3), and mean CK levels (group 1) were up to four times greater than nondiseased fish (groups 5 and 6). These higher CK levels and total inflammation scores were suggested to be the disease (HSMI) outcome and supported the notion that fish included in group 1 were in the acute phase of disease whereas groups 2 and 3 were not in clinical phase of a HSMI outbreak. The acute phase of the disease (HSMI) corresponded to higher mortality rates on the farm and creatine kinase levels in blood sustained over 34 days in humans where a cardiac injury has occurred [28].

The HSMI-infected fish showed significantly higher histopathological scores as compared to the nondiseased fish. The higher histopathological scores were consistent with the higher CK levels in diseased fish as compared to nondiseased fish, supported the higher enzymes levels likely due to myopathy. The total inflammation scores were doubled as compared to the total necrosis scores in all HSMI fish which were considered as clinical sign of HSMI while necrosis being suggested as a secondary effect $[13,15]$. The mean CK and LDH levels and total necrosis scores for chronic CMS fish were equal or lower to the nondiseased fish suggested no correlation to CMS fish and supported the hypothesis that increased enzymes levels identified in the HSMI fish were related to myopathy [30,31]. The fish cages that experienced the CMS outbreak showed high inflammation scores as compared to other cages on the same farm that were not diagnosed with CMS, and both CMS and non-CMS fish had higher values of enzymes indicating that CK and LDH were not correlated to the chronic CMS fish histopathology scores.

The significantly positive correlations were identified with biochemical enzymes (CK and LDH) and histopathological changes in HSMI-affected Atlantic salmon [12, 24]. The histopathological scores for anatomically distinct areas of heart and skeletal muscle were correlated significantly with respective $\mathrm{CK}$ and $\mathrm{LDH}$ levels of the fish $(P=0.05)$. The serum enzymes (CK and $\mathrm{LDH}$ ) correlations have been used previously to find significant relationships in great sturgeon (Huso huso) and rainbow trout (Oncorhynchus mykiss) [24, $37,38]$. The correlations between histopathology (inflammation and necrosis) and enzymes (CK and LDH) values were significant and positive, and suggested the HSMI disease 
effects on blood biochemistry of Atlantic salmon and consistent with mammalian studies where blood biochemistry is changed in pathological conditions and used to predict the disease $[29,36]$. The correlation of CK levels to HSMI histopathology appeared useful due to the release of CK after tissue injury and potential contribution in limited piscine blood biochemistry [28]. However, the CMS group showed non-significant correlations between histology and enzymes and suggested that blood biochemistry of Atlantic salmon might not be affected due to chronic CMS disease. Another reason for no correlation of chronic CMS fish might include the time of fish sampling which was conducted 6 months after acute phase of disease while biochemical enzymes (CK and $\mathrm{LDH}$ ) release rapidly following tissue injury with peak levels for 10-12 days in human [28]. It was also supported by the fact that chronic CMS fish did not show signs (higher mortality levels) and severe histopathological lesions characteristic of acute phase of disease suggesting the late or chronic phase of disease. However, further studies are required to completely understand the CMS disease effects on the blood biochemistry. Histopathology is still a diagnostic method of choice in clinical case of HSMI even though the recent identification of a reovirus associated with the disease allows the possibility of identifying infected animals. However, asymptomatic Atlantic salmon have been identified with piscine reovirus and piscine myocarditis virus by RT-qPCR $[17,25]$. However, it is a terminal procedure performed after the onset of disease and there has been as observed increase mortality level on farm, and mis-diagnosis may result with diseases such as PD and CMS presenting similar pathological changes to HSMI [12]. The blood biochemistry tests may prove useful and have been proved useful in the detection and diagnosis of metabolic disturbances in a number of diseases [39]. The use of serum enzymes to diagnose the cardiac diseases in humans is a common practice and a well-established method. The $\mathrm{CK}$ and LDH levels are affected in the cardiac diseases and serve as disease indicators in humans [26]. Previous attempts at measuring $\mathrm{CK}$ values were made in similar disease such as pancreas disease (PD) but were not correlated directly and extensively with histopathology [30, 31]. These studies have been shown the significantly increased CK levels and suggested the tissue damage as source of enzyme. HSMI fish exhibited higher mean histopathology scores and enzymes (CK and LDH) levels, and serum enzymes showed significant positive correlations to histopathology which supported the notion that CK and LDH levels were affected due to natural HSMI outbreak. The use of CK and LDH enzymes haematological levels for pathological changes at least for HSMI appeared promising and a potential contribution in the limited piscine blood biochemistry by identifying the enzyme ranges in the above-mentioned fish groups [22].

In conclusion, the present study measured the CK and $\mathrm{LDH}$ levels in diseased and nondiseased Atlantic salmon and correlated significantly to the histopathology of Atlantic salmon affected with natural HSMI outbreaks while being non-significant to chronic CMS. The significantly higher CK levels correlated positively and significantly to HSMI pathological changes, suggesting that the potential use of serum enzymes for screening HSMI is promising. The findings of the present study should be considered as a contribution to the more extensive research necessary to understand biological activities (enzymes) and the pathological changes of Atlantic salmon.

\section{Acknowledgments}

The authors would like to thank Anil Amin for assistance in histology, Dr. Silvia Spirova, Dr. Erik Wilkinson, Ørjan Wenberg, Dr. Johan Johansen, and Kristin Dahlen for providing samples and Thoresen Stein Istre and Ane Lise Mohus for enzyme analysis.

\section{References}

[1] T. T. Poppe and H. W. Ferguson, "Cardiovascular system," in Systemic Pathology of Fish: A Text and Atlas of Normal Tissue Responses in Teleosts, and Their Responses in Disease, H. W. Ferguson, Ed., pp. 141-167, Scotian Press, London, UK, 2006.

[2] T. T. Poppe, T. Taksdal, and P. H. Bergtun, "Suspected myocardial necrosis in farmed Atlantic salmon, Salmo salar L.: a field case," Journal of Fish Diseases, vol. 30, no. 10, pp. 615-620, 2007.

[3] A. P. Farrell, "Cardiorespiratory performance in salmonids during exercise at high temperature: insights into cardiovascular design limitations in fishes," Comparative Biochemistry and Physiology A, vol. 132, no. 4, pp. 797-810, 2002.

[4] T. T. Poppe and T. Taksdal, "Ventricular hypoplasia in farmed Atlantic salmon Salmo salar," Diseases of Aquatic Organisms, vol. 42, no. 1, pp. 35-40, 2000.

[5] H. W. Ferguson, T. T. Poppe, and D. J. Speare, "Cardiomyopathy in farmed Norwegian salmon," Diseases of Aquatic Organisms, vol. 8, pp. 225-231, 1990.

[6] S. Grotmol, G. K. Totland, and H. Kryvi, "Detection of a nodavirus-like agent in heart tissue from reared Atlantic salmon Salmo salar suffering from cardiac myopathy syndrome (CMS)," Disease of Aquatic Organisms, vol. 29, no. 2, pp. 7984, 1997.

[7] E. Brun, T. T. Poppe, A. Skrudland, and J. Jarp, "Cardiomyopathy syndrome in farmed Atlantic salmon Salmo salar: occurrence and direct financial losses for Norwegian aquaculture," Diseases of Aquatic Organisms, vol. 56, no. 3, pp. 241-247, 2003.

[8] T. Taksdal, A. B. Olsen, I. Bjerkås et al., "Pancreas disease in farmed Atlantic salmon, Salmo salar L., and rainbow trout, Oncorhynchus mykiss (Walbaum), in Norway," Journal of Fish Diseases, vol. 30, no. 9, pp. 545-558, 2007.

[9] R. T. Nelson, M. F. McLoughlin, H. M. Rowley, M. A. Platten, and J. I. McCormick, "Isolation of a toga-like virus from farmed Atlantic salmon Salmo salar with pancreas disease," Diseases of Aquatic Organisms, vol. 22, no. 1, pp. 25-32, 1995.

[10] K. E. Christie, D. A. Graham, M. F. McLoughlin, S. Villoing, D. Todd, and D. Knappskog, "Experimental infection of Atlantic salmon Salmo salar pre-smolts by i.p. injection with new Irish and Norwegian salmonid alphavirus (SAV) isolates: a comparative study," Diseases of Aquatic Organisms, vol. 75, no. 1, pp. 13-22, 2007.

[11] R. T. Kongtorp, A. Kjerstad, T. Taksdal, A. Guttvik, and K. Falk, "Heart and skeletal muscle inflammation in Atlantic salmon, Salmo salar L.: a new infectious disease," Journal of Fish Diseases, vol. 27, no. 6, pp. 351-358, 2004. 
[12] R. T. Kongtorp, M. Halse, T. Taksdal, and K. Falk, "Longitudinal study of a natural outbreak of heart and skeletal muscle inflammation in Atlantic salmon, Salmo salar L.," Journal of Fish Diseases, vol. 29, no. 4, pp. 233-244, 2006.

[13] R. T. Kongtorp, T. Taksdal, and A. Lyngøy, "Pathology of heart and skeletal muscle inflammation (HSMI) in farmed Atlantic salmon Salmo salar," Diseases of Aquatic Organisms, vol. 59, no. 3, pp. 217-224, 2004.

[14] H. W. Ferguson, R. T. Kongtorp, T. Taksdal, D. Graham, and K. Falk, "An outbreak of disease resembling heart and skeletal muscle inflammation in Scottish farmed salmon, Salmo salar L., with observations on myocardial regeneration," Journal of Fish Diseases, vol. 28, no. 2, pp. 119-123, 2005.

[15] R. T. Kongtorp and T. Taksdal, "Studies with experimental transmission of heart and skeletal muscle inflammation in Atlantic salmon, Salmo salar L.," Journal of Fish Diseases, vol. 32, no. 3, pp. 253-262, 2009.

[16] G. Palacios, M. Lovoll, T. Tengs et al., "Heart and skeletal muscle inflammation of farmed salmon is associated with infection with a novel reovirus," PLoS One, vol. 5, no. 7, Article ID e11487, 2010.

[17] C. R. Wiik-Nielsen, P. M. R. Ski, A. Aunsmo, and M. Lovoll, "Prevelance of viral RNA from piscine reovirus and piscine myocarditis virus in Atlantic salmon, Salmo salar L., broodfish and progeny," Journal of Fish Diseases, vol. 35, pp. 169-171, 2012.

[18] H. R. Skjelstad, G. Bornø, Flesjå K. et al., "The health situation in farmed fish in Norway 2007," Report by National Veterinary Institute, Norway, pp. 1-20, 2007.

[19] A. Marta, E. Biering, D. Colquhoun et al., "Helsesituasjonen hos laksefisk 2011 [health situation in farmed Atlantic salmon 2011; in Norwegian]," in Fiskehelserapporten 2011 [Fish Health Report 2011], A. B. Olsen, Ed., pp. 1-32, Report from the Norwegian Veterinary Institute, Norway 2010, 2011.

[20] M. Løvoll, J. Wiik-Nielsen, S. Grove et al., "A novel totivirus and piscine reovirus (PRV) in Atlantic salmon (Salmo salar) with cardiomyopathy syndrome (CMS)," Virology Journal, vol. 7, article 309, 2010.

[21] Ø. Haugland, A. B. Mikalsen, P. Nilsen et al., "Cardiomyopathy syndrome of Atlantic salmon (Salmo salar L.) is caused by a double-stranded RNA virus of the Totiviridae family," Journal of Virology, vol. 85, no. 11, pp. 5275-5286, 2011.

[22] M. D. Powell, "Physiological and clinical pathology," in Systemic Pathology of Fish: A Text and Atlas of Normal Tissue Responses in Teleosts, and Their Responses in Disease, H. W. Ferguson, Ed., pp. 336-356, Scotian Press, London, UK, 2006.

[23] K. Sandnes, O. Lie, and R. Waagbo, "Normal ranges of some blood chemistry parameters in adult farmed Atlantic salmon, Salmo salar," Journal of Fish Biology, vol. 32, no. 1, pp. 129136, 1988.

[24] C. Y. Chen, G. A. Wooster, and P. R. Bowser, "Comparative blood chemistry and histopathology of tilapia infected with Vibrio vulnificus or Streptococcus iniae or exposed to carbon tetrachloride, gentamicin, or copper sulfate," Aquaculture, vol. 239, no. 1-4, pp. 421-443, 2004.

[25] A. H. Garseth, M. Opheim, C. Fristvold, E. Skjerve, and E. Biering, Piscine reovirus (PRV) in wild Atlantic salmon, Salmo salar L. and sea trout, Salmo trutta in Norway, Abstract 15th EAFP, Prague, Czech Republic, [O-0149], 2011.

[26] F. S. Apple, M. Murakami, M. Panteghini et al., "International survey on the use of cardiac markers," Clinical Chemistry, vol. 47 , no. 3 , pp. $587-588,2001$.
[27] D. B. Walker, "Serum chemical biomarkers of cardiac injury for nonclinical safety testing," Toxicologic Pathology, vol. 34, no. 1, pp. 94-104, 2006.

[28] M. Kemp, J. Donovan, H. Higham, and J. Hooper, "Biochemical markers of myocardial injury," British Journal of Anaesthesia, vol. 93, no. 1, pp. 63-73, 2004.

[29] Y. Ishikawa, J. E. Saffitz, T. L. Mealman, A. M. Grace, and R. Roberts, "Reversible myocardial ischemic injury is not associated with increased creatine kinase activity in plasma," Clinical Chemistry, vol. 43, no. 3, pp. 467-475, 1997.

[30] H. D. Rodger, T. M. Murphy, E. M. Drinan, and D. A. Rice, "Acute skeletal myopathy in farmed Atlantic salmon Salmo salar," Diseases of Aquatic Organisms, vol. 12, pp. 17-23, 1991.

[31] H. W. Ferguson, D. A. Rice, and J. K. Lynas, "Clinical pathology of myodegeneration (pancreas disease) in Atlantic salmon (Salmo salar)," The Veterinary Record, vol. 119, no. 12, pp. 297299, 1986.

[32] R. T. Kongtorp, Heart and skeletal muscle inflammation (HSMI) in Atlantic salmon, Salmo salar: pathology, pathogenesis and experimental infection, Ph.D. thesis, Unipub AS Oslo, 2008.

[33] M. Burke, Effects of simulated anaemia on the blood chemistry of Atlantic cod, Atlantic halibut and Atlantic salmon, Master thesis, Bodø University College, 2009.

[34] M. F. McLoughlin, D. A. Graham, A. Norris et al., "Virological, serological and histopathological evaluation of fish strain susceptibility to experimental infection with salmonid alphavirus," Diseases of Aquatic Organisms, vol. 72, no. 2, pp. 125-133, 2006.

[35] L. Greco, E. Capri, and T. Rustad, "Biochemical responses in Salmo salar muscle following exposure to ethynylestradiol and tributyltin," Chemosphere, vol. 68, no. 3, pp. 564-571, 2007.

[36] J. A. Almeida, Y. S. Diniz, S. F. G. Marques et al., "The use of the oxidative stress responses as biomarkers in Nile tilapia (Oreochromis niloticus) exposed to in vivo cadmium contamination," Environment International, vol. 27, no. 8, pp. 673-679, 2002.

[37] Z. H. Li, J. Velisek, R. Grabic, P. Li, J. Kolarova, and T. Randak, "Use of hematological and plasma biochemical parameters to assess the chronic effects of a fungicide propiconazole on a freshwater teleost," Chemosphere, vol. 83, no. 4, pp. 572-578, 2011.

[38] F. Rajabipour, D. Shahsavani, A. Moghimi, S. Jamili, and N. Mashaii, "Comparison of serum enzyme activity in great sturgeon, Huso huso, cultured in brackish and freshwater earth ponds in Iran," Comparative Clinical Pathology, vol. 19, no. 3, pp. 301-305, 2010.

[39] E. S. Celik, "Blood chemistry (electrolytes, lipoproteins and enzymes) values of black scorpion fish (Scorpaena porcus Linneaus, 1758) in the Dardanelles," Turkey Journal of Biological Sciences, vol. 4, pp. 716-719, 2004. 


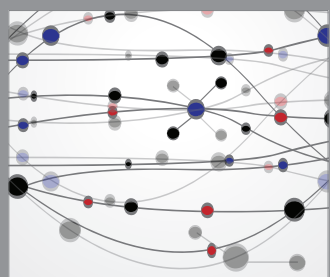

The Scientific World Journal
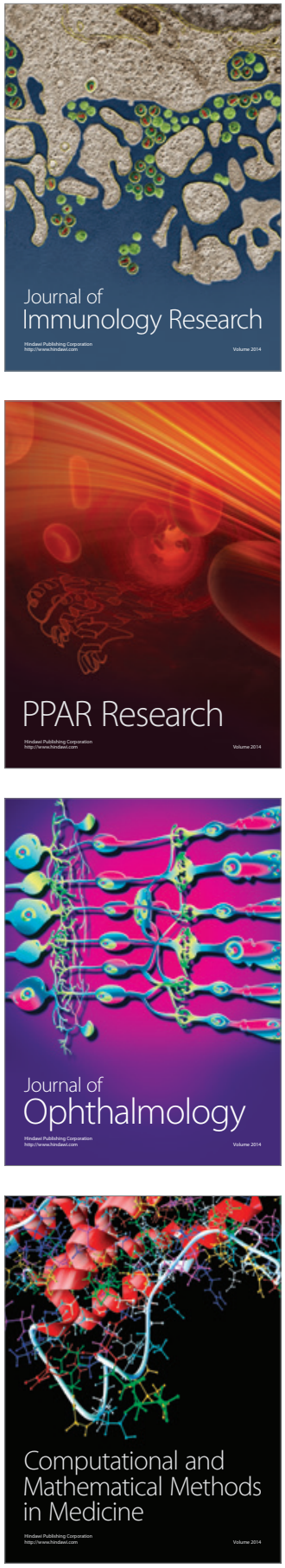

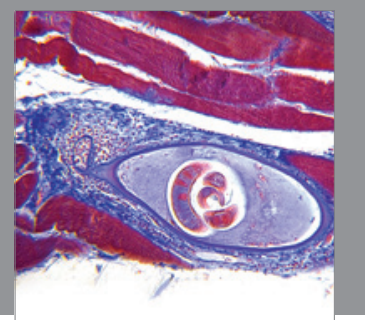

Gastroenterology

Research and Practice
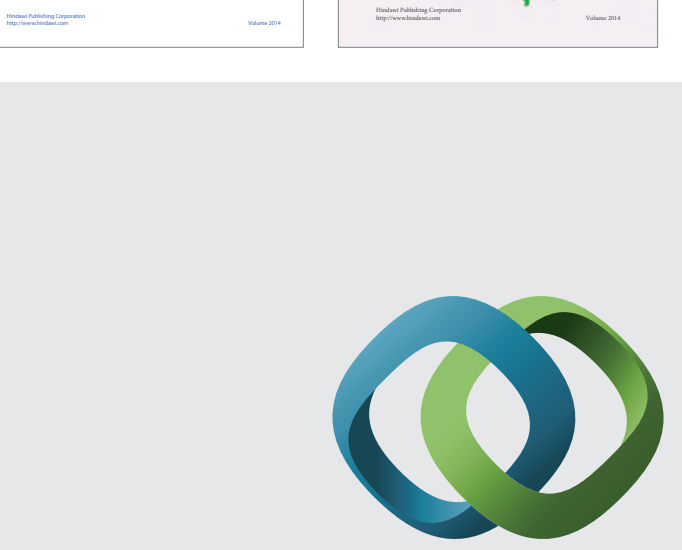

\section{Hindawi}

Submit your manuscripts at

http://www.hindawi.com
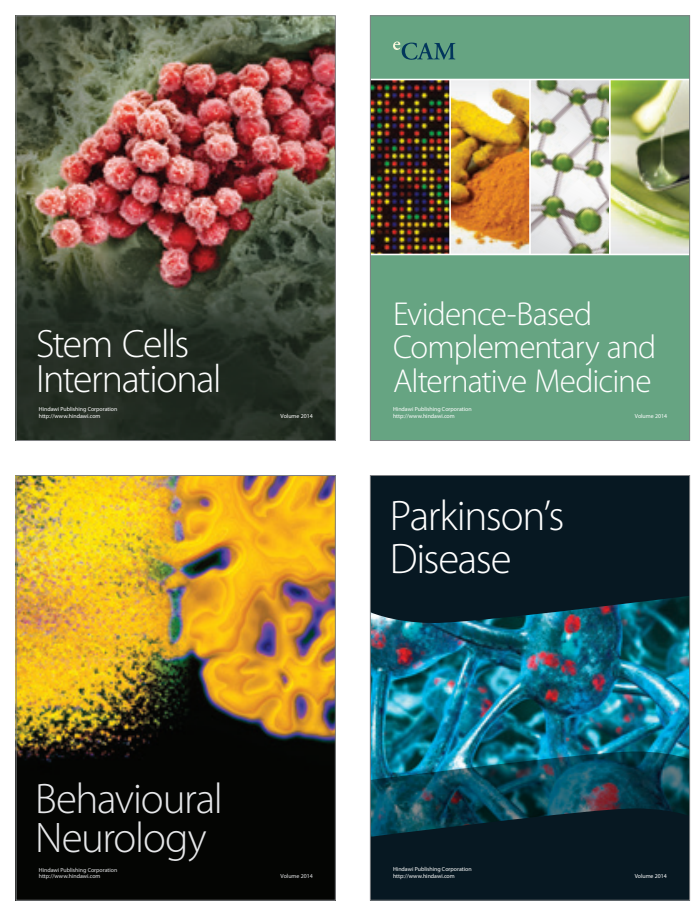

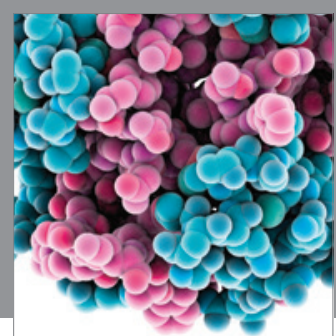

Journal of
Diabetes Research

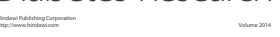

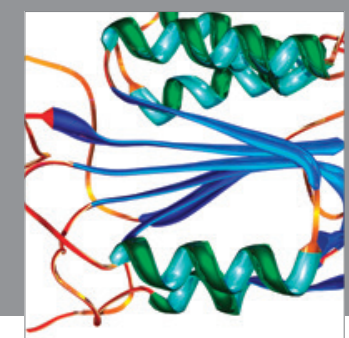

Disease Markers
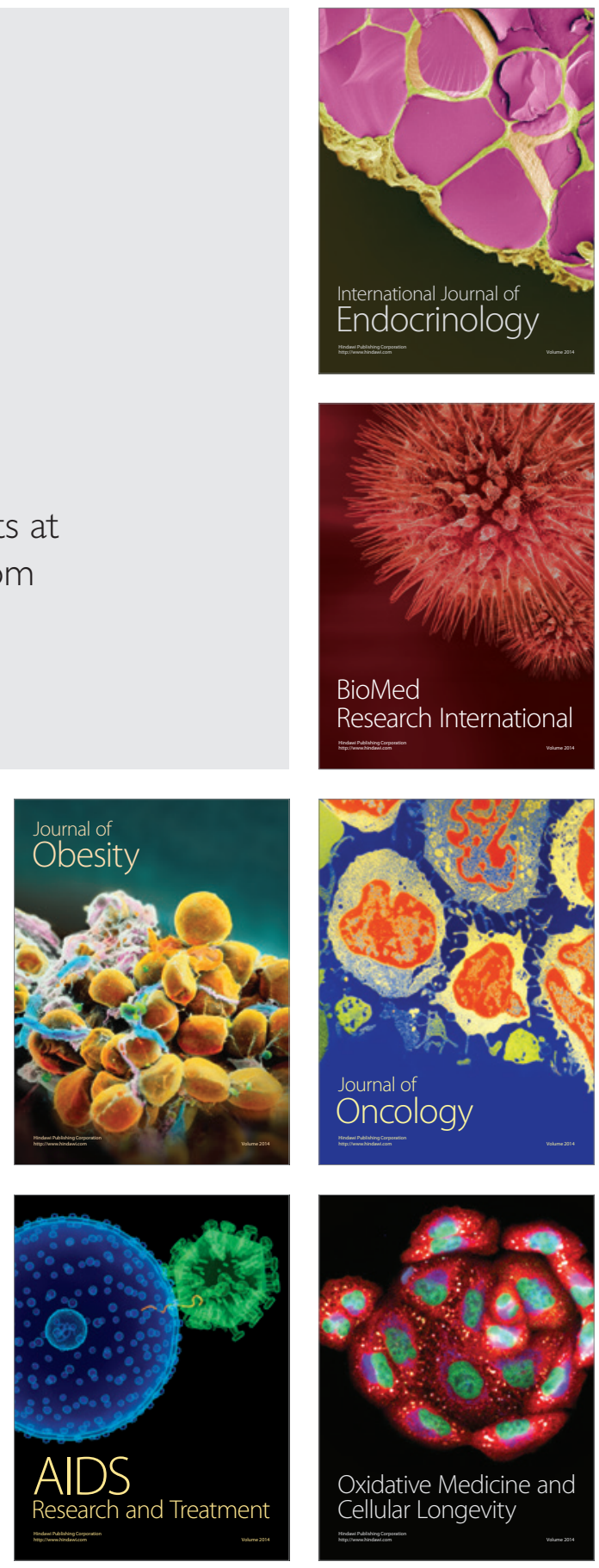